\title{
Etude expérimentale de l'écoulement instationnaire dans le diffuseur aubé d'une turbomachine centrifuge
}

\author{
Experimental investigation of the unsteady flow in a centrifugal \\ turbomachine vaned diffuser
}

par Marina Ubaldi, Pietro Zunino, Andrea Cattanei

Istituto di Macchine e Sistemi Energetici — Università di Genova (Italie)

The unsteady effects caused by the interaction of the rotor wakes with the flow within the vaned diffuser of a centrifugal turbomachine stage have been experimentally investigated.

The experiment has been carried out on the simplified model of a centrifugal pump operating in air at the nominal point. The diffuser flow has been surveyed by means of a two-colour four-beam laser Doppler velocimeter on a blade-to-blade surface at midspan and over two cylindrical measuring surfaces located just upstream of the diffuser vanes leading edges and in proximity of the inlet throat of the diffuser. Instantaneous data taken in phase with the shaft rotation have been averaged and used to construct images of the flow field for different relative positions of impeller blades and diffuser vanes. The sequences are detailed enough to show the evolution of the rotor wakes within the forward part of the diffuser. The rotor blade wakes entering the diffuser are chopped by the vanes leading edges into individual segments which are convected through the diffuser passages where they mix out rapidly.

\section{INTRODUCTION}

L'écoulement dans les turbomachines est intrinsèquement instationnaire à cause de l'interaction aérodynamique entre les composants fixes et tournants en mouvement relatif. Ce phénomène affecte les performances aérodynamiques et le comportement acoustique et vibratoire de la machine.

L'interaction aérodynamique se réalise par deux mécanismes: le premier est généré par le déplacement relatif des champs de mouvement non-uniformes qui sont engendrés dans les deux rangées d'aubage adjacentes et affecte soit la grille amont soit la grille aval (effet à potentiel); le second mécanisme est dû à l'interaction des sillages des aubes de la grille amont avec les aubes de la grille aval.

Dans les turbomachines centrifuges, à cause des faibles entrefers entre roue et diffuseur et du mélange rapide des sillages, les deux mécanismes sont comparables. L'effet à potentiel induit par le diffuseur aubé sur l'écoulement dans la roue est limité à la zone de sortie et diminue rapidement à l'intérieur des canaux de la roue, mais il n'est pas négligeable, car il influence la répartition azimutale du travail spécifique et de la vitesse débitante et peut être la cause de décollements locaux de l'écoulement dans la roue $[1,2]$.

En ce qui concerne l'effet de l'écoulement du rotor sur le diffuseur aubé, les relevés expérimentaux menés sur des compresseurs et des pompes centrifuges [1-5] montrent, pour les sections de sondage situées à l'entrée du diffuseur, un écoulement fortement instationnaire. Cet écoulement est caractérisé par des variations élevées de la vitesse à la fréquence de passage des aubes de la roue, qui sont indicatives de sillages d'aubage ou de structures d'écoulement de type sillage-jet pas encore mélangés. En aval de la section minimale d'entrée du diffuseur, les fluctuations de vitesse décroissent significativement, en montrant que le mélange des non-uniformités circonférentielles se réalise rapidement dans le diffuseur $[3,5]$. Cela ne signifie pas que l'effet de l'interaction entre rotor et stator soit limité à la zone d'entrée du diffuseur: en effet les fluctuations de pression semblent se propager plus profondément dans le diffuseur et, d'un autre côté, on peut supposer que la couche limite sur les aubes du diffuseur, en analogie avec les relevés expérimentaux menés en étages de turbomachines axiales $[6,7]$, soit fortement influencée par le passage alterné d'un écoulement caractérisé par des niveaux de turbulence très différents.

Récemment 1'utilisation de techniques de mesure du champ de mouvement instantané, telle que la Particle Image Velocimetry [5], a permis d'étendre l'investigation sur un plan aube-à-aube complet du diffuseur, en mettant en évidence la structure tourbillonnante de l'écoulement dans la zone d'entrée. Cependant, pour le cas d'étages centrifuges, les résultats expérimentaux suffisamment détaillés dans l'espace pour permettre l'étude du mélange des sillages de la roue dans le diffuseur aubé sont peu nombreux. 
Le travail présenté dans cet article est une contribution à ce type d'approche au problème. On reporte les résultats d'une investigation expérimentale sur le développement des sillages de la roue dans le diffuseur aubé d'un étage de turbomachine centrifuge. Ces résultats représentent une intégration à une base de données expérimentales déjà disponible concernant l'étude de l'effet du diffuseur aubé sur l'écoulement dans la roue $[2,8]$. L'ensemble de ces données constitue un cas-test bien documenté sur l'interaction aérodynamique rotor-stator dans un étage centrifuge.

\section{II — INSTALLATION D'ESSAIS}

Les essais ont été effectués sur un modèle de turbomachine centrifuge, constitué par une roue entièrement radiale sans couvercle et un diffuseur aubé (fig. 1). Le modèle fonctionne dans un circuit ouvert en air, en prélevant le fluide à conditions atmosphériques par une conduite d'aspiration munie d'un nid d'abeille et une vanne pour modifier le point de fonctionnement. L'écoulement est rejeté en sortie du diffuseur aubé directement dans l'atmosphère sans l'utilisation d'une volute.

La roue de diamètre $420 \mathrm{~mm}$ est constituée de 7 aubes à simple courbure, couchées en arrière. Le diffuseur est muni

\section{NOMENCLATURE \\ $b \quad$ largeur de la roue et du diffuseur \\ c vitesse absolue \\ $G_{d} \quad$ pas circonférentiel des aubes du diffuseur \\ $G_{i} \quad$ pas circonférentiel des aubes de la roue \\ $f \quad$ fréquence de passage de l'aubage \\ $p_{t} \quad$ pression totale \\ $Q \quad$ débit volumétrique \\ $r \quad$ coordonnée radiale \\ $R_{2} \quad$ rayon en sortie de roue \\ $t \quad$ temps \\ $T_{i} \quad$ période de passage des aubes de la roue \\ $T u \quad$ taux de turbulence $\left[\left(\tilde{C_{r}^{12}}+\tilde{C}_{u}^{12}\right) / 2\right]^{1 / 2} / U_{2}$ \\ $U_{2} \quad$ vitesse d'entraînement en sortie de roue \\ $y \quad$ coordonnée en direction azimutale \\ $z \quad$ coordonnée axiale \\ $z_{d} \quad$ nombre d'aubes du diffuseur \\ $z_{i} \quad$ nombre d'aubes de la roue \\ $\rho$ masse volumique \\ $\varphi \quad$ coefficient de débit $=Q /\left(U_{2} \pi R_{2}{ }^{2}\right)$ \\ $\psi \quad$ coefficient de pression totale $=$ \\ $2\left(\mathrm{p}_{t 4}-p_{t 0}\right) /\left(\rho U_{2}^{2}\right)$ \\ $\omega \quad$ vitesse angulaire de rotation}

\section{INDICES}

$r \quad$ en direction radiale

$u \quad$ en direction tangentielle

0 dans la conduite d'aspiration

2 en sortie de roue

3 à l'entrée du diffuseur

4 en sortie de diffuseur

, fluctuation

$\sim \quad$ moyenne en phase

- moyenne suivant le temps

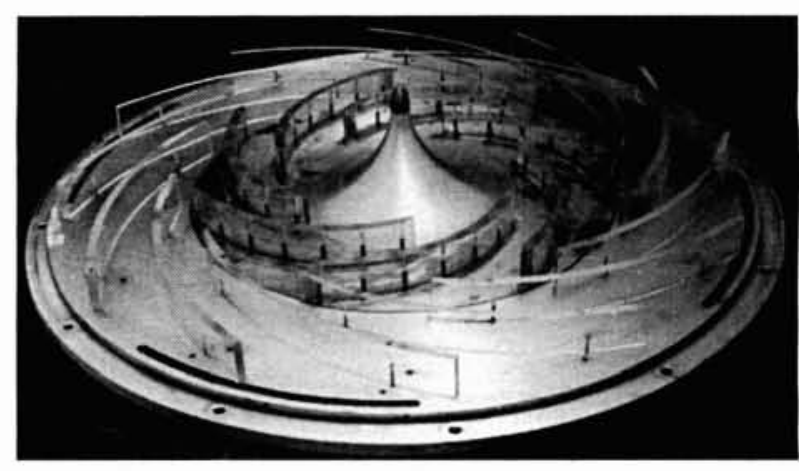

1. Roue centrifuge et diffuseur aubé.

de 12 aubes, dont les bords d'attaque sont situés à une distance radiale de $6 \%$ des bords de fuite des aubes de la roue. La largeur de la roue et du diffuseur est constante $(b=40 \mathrm{~mm})$. Les coordonnées des aubes de la roue et du diffuseur sont données dans [2].

Les données géométriques des canaux interaubes du rotor et du stator déterminent des rapports de diffusion géométrique respectivement de 1,42 et 2,03. Ces valeurs placent les canaux des deux composants de la machine proche de la ligne qui sépare les zones de décollement non-appréciable et décollement de coin sur le diagramme des performances des diffuseurs bidimensionnels tournants de Rothe et Johnston [9].

Les principales données géométriques du modèle sont résumées dans le tableau 1 .

Les essais ont été effectués au régime de fonctionnement nominal, défini par le coefficient de débit $\varphi=0,048$ et le coefficient de hauteur $\psi=0,65$, et à la vitesse de rotation de $2000 \mathrm{tr} / \mathrm{min}$.

La paroi carter du modèle est instrumentée avec des prises de pression, des capteurs de pression à réponse rapide, des supports pour sondes à fil chaud et un hublot en verre donnant l'accès aux canaux de la roue et du diffuseur pour mesures laser (ALD).

\section{III — INSTRUMENTATION ET TECHNIQUE DE MESURE}

Les mesures ont été effectuées à l'aide d'un vélocimètre laser à effet Doppler à 2 réseaux de franges d'interférence,

Tableau 1. - Données géométriques et conditions de fonctionnement

Roue

diamètre d'entrée

diamètre de sortie

largeur d'aubage

nombre d'aubes

angle d'entrée d'aubage

angle de sortie d'aubage

\section{Diffuseur}

diamètre d'entrée diamètre de sortie

largeur d'aubage

nombre d'aubes

angle d'entrée d'aubage

angle de sortie d'aubage

\section{Conditions de}

fonctionnement

vitesse de rotation

coefficient de débit

coefficient de pression totale
$D_{1}=240 \mathrm{~mm}$

$D_{2}=420 \mathrm{~mm}$

$b=40 \mathrm{~mm}$

$z_{i}=7$

$\beta_{i}^{\prime}=-65^{\circ}$

$\beta_{2}^{\prime}=-70^{\circ}$

$D_{3}==444 \mathrm{~mm}$

$D_{4}=664 \mathrm{~mm}$

$b=40 \mathrm{~mm}$

$z_{d}=12$

$\alpha_{3}^{\prime}=74^{\circ}$

$\alpha_{4}^{\prime}=68^{\circ}$

$n=2000 \mathrm{tr} / \mathrm{min}$

$\varphi=0,048$

$\psi=0,65$ 
équipé par un laser à argon (puissance $300 \mathrm{~mW}$ ) et une sonde à fibres optiques pour le transport des faisceaux incidents et de la lumière rétrodiffusée.

On a utilisé une lentille frontale de diamètre $60 \mathrm{~mm}$ avec longueur focale de $160 \mathrm{~mm}$, qui donne lieu à un volume de mesure de diamètre $0,08 \mathrm{~mm}$ et longueur $0,64 \mathrm{~mm}$. Le fluide a été ensemencé à l'entrée de la conduite d'aspiration par un spray atomisé de particules d'huile minérale (diamètre 0,5$2 \mu \mathrm{m})$. Les signaux Doppler ont été traités par deux analyseurs de spectre (Burst Spectrum Analysers).

On a enregistré 200000 données pour chaque point de mesure. L'acquisition simultanée des temps d'arrivée des signaux Doppler et d'un signal synchronisé sur le passage d'une aube de la roue a permis de grouper les données sur 420 fenêtres angulaires ( 60 pour chaque canal de la roue).

Ces données ont été moyennées en phase pour séparer la part périodique du signal due à la non-uniformité spatiale de l'écoulement en sortie de roue et les fluctuations aléatoires dues à la turbulence. Les équations suivantes définissent la vitesse moyennée en phase et la valeur r.m.s. des fluctuations associées à tous les phénomènes instationnaires qui ne sont pas corrélés avec la fréquence de rotation de la roue :

$$
\begin{aligned}
& \tilde{c}(j, k)=\frac{1}{N} \sum_{n=1}^{N} c(j, n, k) \\
& \sqrt{C^{\tilde{1} 2}(j, k)}=\sqrt{\frac{1}{N} \sum_{n=1}^{N}[c(j, n, k)-\tilde{c}(j, k)]^{2}}
\end{aligned}
$$

où $c(j, n, k)$ est la vitesse instantanée mesurée dans la fenêtre angulaire $j$, pendant l'enregistrement $n$, pour la position relative $k$ entre le volume de mesure et le diffuseur. $N$ est le nombre total des données enregistrées dans la fenêtre $j$.

Pour chaque point de mesure, en moyennant la vitesse $\tilde{c}(j, k)$ suivant le temps, on peut obtenir une vitesse $\overline{\tilde{c}}(k)$ qui ne dépend pas de la position relative roue-diffuseur

$$
\tilde{c}(k)=\sum_{j=1}^{J} \tilde{c}(j, k) / J
$$

et la valeur r.m.s. des fluctuations autour de cette moyenne représente l'instationnarité générée par la roue

$$
\sqrt{\tilde{\bar{c}}^{2}(k)}=\sqrt{\sum_{j=1}^{J}[\tilde{c}(j, k)-\bar{c}(k)]^{2} / J}
$$

\section{IV — INVESTIGATION EXPÉRIMENTALE SUR L'INTERACTION DE L'ÉCOULEMENT EN SORTIE DE ROUE AVEC LE DIFFUSEUR AUBÉ}

L'interaction de l'écoulement en sortie de roue avec le diffuseur aubé a été étudiée en analysant la zone d'entrée du diffuseur sur un plan aube-à-aube à mi-largeur et sur deux surfaces cylindriques circonférentielles.

Le domaine de mesure dans le plan aube-à-aube s'étend en direction radiale à partir de $r / R_{2}=0,96$ jusqu'à $r / R_{2}=1,26$ (rapport $R_{3} / R_{2}$ sur le bord d'attaque des aubes du diffuseur $=1,06$ ) et il est composé de 32 points de mesure en direction radiale et 24 en direction azimutale sur un pas du diffuseur.

La première surface de mesure cylindrique est située juste en amont du bord d'attaque des aubes du diffuseur $\left(r / R_{2}=1,048\right)$, la seconde à $r / R_{2}=1,095$ coupe la section d'entrée minimale du diffuseur. Chaque surface est définie par 24 points en direction azimutale sur un pas du diffuseur et 15 points en direction axiale entre $z / b=0,075$ et $z / b=0,925(z / b=0$ au moyeu et $z / b=1$ au carter).

Pour chaque point de mesure les composantes instantanées de la vitesse en direction radiale et azimutale ont été enregistrées simultanément. Les données obtenues ont été traitées à l'aide des techniques décrites dans le paragraphe précédent.

Les données moyennées en phase pour les différentes positions azimutales ont été interpolées pour obtenir des images instantanées de l'écoulement pour différentes positions des aubes de la roue par rapport au diffuseur. Ces images montrent les mécanismes d'interaction instationnaire de l'écoulement en sortie de roue avec les aubes du diffuseur.

\section{- 4.1 Répartition de l'écoulement sur les surfaces cylindriques}

Les figures 2 et 3 montrent sur les surfaces cylindriques de mesure $r / R_{2}=1,048$ et $r / R_{2}=1,095$ les répartitions du module de la vitesse absolue $\tilde{c}$ moyennée en phase et du taux de turbulence $\widetilde{T u}$, évalué à partir des fluctuations des deux composantes de la vitesse mesurées. Les isocontours sont représentés en fonction de la coordonnée circonférentielle réduite $y / G_{i}, G_{i}$ étant le pas circonférentiel local du rotor $G_{i}=2 \pi r / z_{i}$, et de la coordonnée axiale réduite $z / b, b$ étant la largeur d'aubage. L'échelle de représentation dans la direction axiale est dilatée par rapport à l'échelle physique par un facteur 2, pour assurer une meilleure lisibilité des variations en cette direction.

Pour des raisons de limitation d'espace, on présente ces graphiques seulement pour un instant de temps $\left(t / T_{i}=0,292\right)$ et plusieurs pas du rotor et du stator. Puisque le nombre des aubes du rotor $\left(z_{i}=7\right)$ et celui des aubes du diffuseur $\left(z_{d}=12\right)$ sont différents, un seul instant suffit pour montrer plusieurs positions relatives entre les canaux du rotor et du stator.

Sur les graphiques les aubes de la roue se déplacent de la droite vers la gauche. Les aubes du diffuseur sont localisées par les bandes verticales dépourvues de valeurs mesurées, dues à l'intersection des rayons laser avec les aubes du diffuseur. Le côté en pression de l'aube du stator est situé sur la droite par rapport à la zone où la mesure est entrecoupée.

Les graphiques de figure 2 montrent que le champ de mouvement est caractérisé par l'alternance dans la direction azimutale de conditions d'écoulement à faible et haute vitesse, associées au passage des sillages de la roue. Les variations de vitesse sont plus élevées à l'entrée du diffuseur $r / R_{2}=1,048$ (valeurs de $\tilde{c} / U_{2}$ comprises entre 0,25 et 0,45 ) et décroissent, bien qu'elles restent encore importantes, dans la section de mesure à $r / R_{2}=1,095$ (valeurs comprises entre 0,35 et 0,45 ). L'augmentation de la vitesse absolue dans les zones perturbées par le sillage est due au défaut de vitesse relative, au glissement de l'écoulement relatif qui est plus faible dans le sillage et à la géométrie de la roue avec aubes couchées en arrière.

La répartition de la vitesse absolue dépend aussi de l'effet à potentiel induit par les aubes du diffuseur. Sur la surface à $r / R_{2}=1,048$ la vitesse diminue près de la ligne de stagnation, sur la droite des bandes verticales dépourvues de données. Sur la surface à $r / R_{2}=1,095$ on peut remarquer près du côté pression de l'aube une augmentation localisée de la vitesse, due à une faible incidence négative de l'écoulement absolu sur les aubes du diffuseur.

Le taux de turbulence est l'un des paramètres qui permettent de localiser plus aisément les sillages des aubes de la roue dans l'écoulement non-uniforme et instationnaire du diffuseur. Sur la figure 3, surface de mesure $r / R_{2}=1,048$, la position et l'étendue azimutale du sillage sont mises en évidence clairement par les bandes verticales sombres, caracté- 


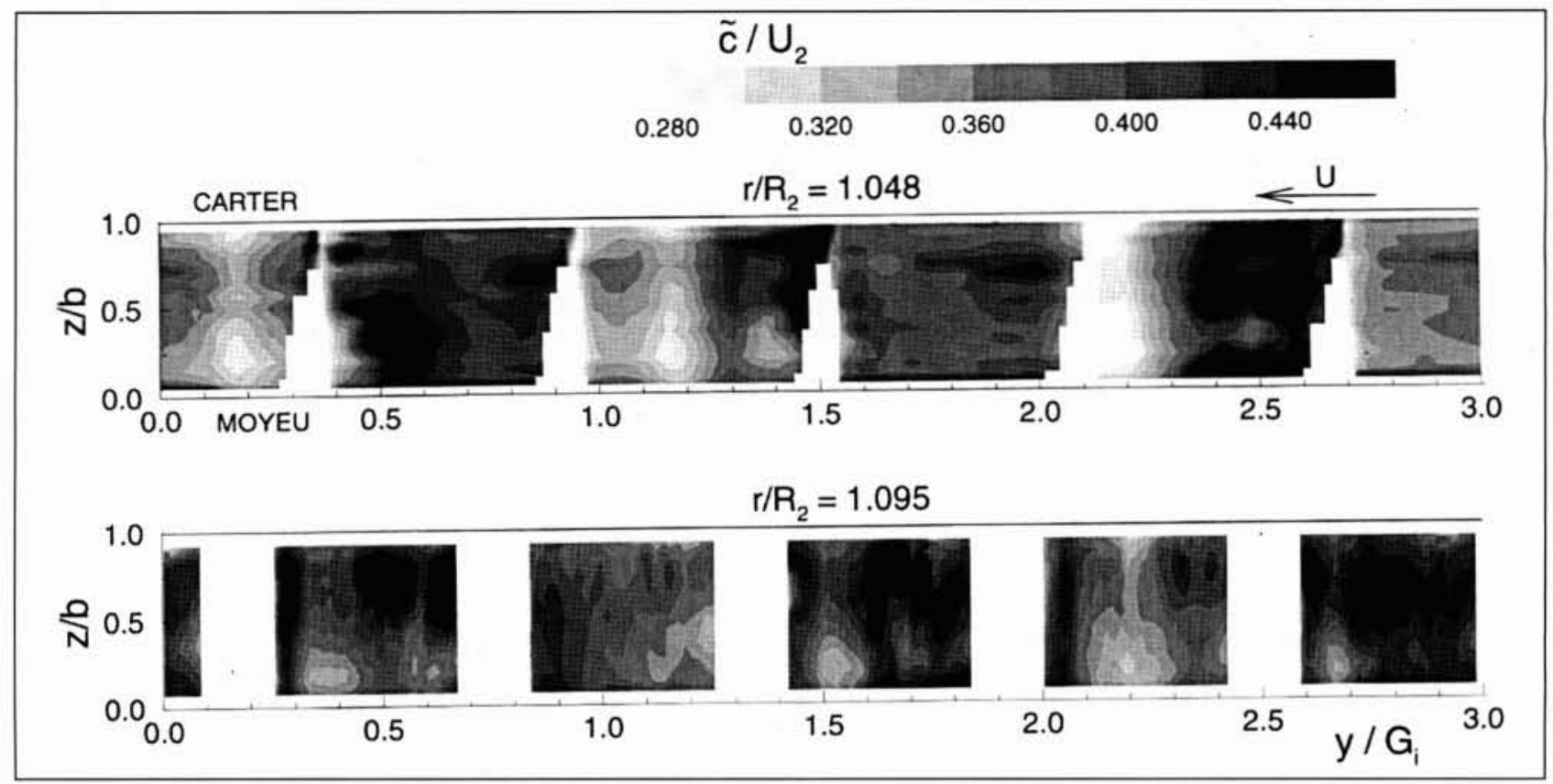

2. Répartitions instantanées de la vitesse absolue sur les surfaces de mesure cylindriques.

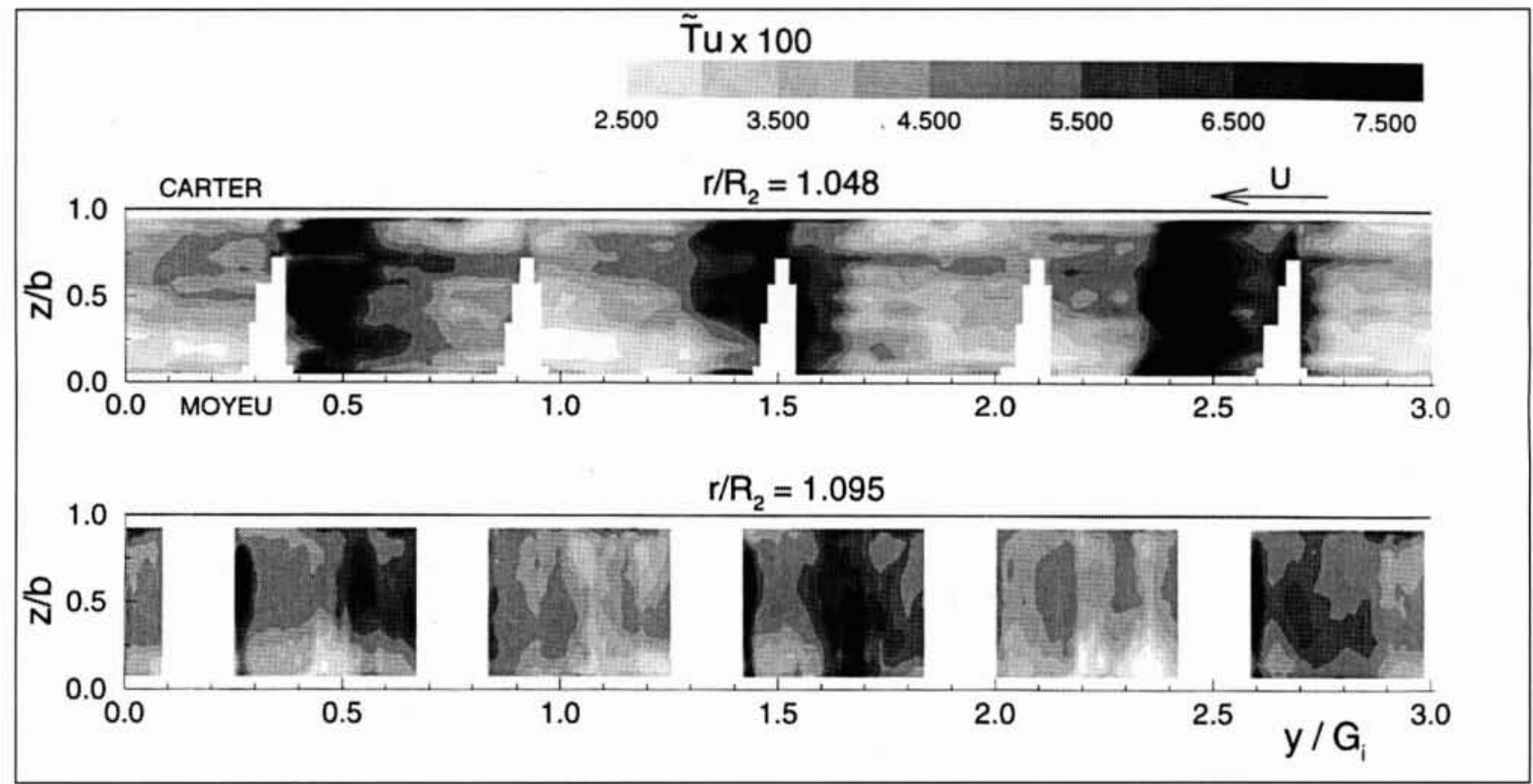

3. Répartitions instantanées du taux de turbulence sur les surfaces de mesure cylindriques.

risées par un taux de turbulence, rapporté à la vitesse d'entraînement $U_{2}$, variable entre 7 et $10 \%$. Si on rapporte le taux de turbulence à la vitesse absolue, ces valeurs approximativement redoublent. On peut supposer, en outre, que l'alternance d'écoulement très turbulent et à faible turbulence, montrée par le graphique, influence le développement de la couche limite sur les aubes du diffuseur.

Les valeurs les plus faibles de turbulence, comprises entre 2 et $3 \%$, sont localisées près du moyeu, dans les zones non perturbées par les sillages. Des valeurs plus élevées, autour de $5 \%$, situées dans une zone proche du côté pression du sillage d'aubage et déplacée vers le carter peuvent être attribuées à un phénomène de sillage qui s'engendre dans le passage interaubes de la roue par l'action des écoulements secondaires et du jeu entre aube et carter.
Sur la seconde section de mesure $\left(r / R_{2}=1,095\right)$, le processus de mélange par action convective et diffusive a atténué le niveau de turbulence dans le sillage, en augmentant en même temps le niveau dans les zones adjacentes. De cette façon le sillage paraît plus étendu, mais moins discernable. En outre, au moment du passage du sillage dans le canal du diffuseur, sur le côté pression de l'aube le taux de turbulence atteint des niveaux très élevés (supérieurs à $8 \%$ ) sur une zone étroite qui s'étend le long de la largeur de l'aubage. On peut penser que cette augmentation de la turbulence est un effet direct de l'action convective de l'écoulement dans le sillage. En effet, selon le modèle proposé par Meyer [10], le sillage d'une aube de rotor, dans le repère du stator, peut être représenté en superposant un jet à l'écoulement moyen non perturbé. La direction du jet dépend du type de grille consi- 
déré. Dans les étages de compresseur, soit axiaux soit centrifuges, le jet est dirigé du côté dépression vers le côté pression du canal du diffuseur. La turbulence comme les autres propriétés du fluide est transportée au travers du canal interaubes vers la surface en pression de l'aubage.

\subsection{Répartition de l'écoulement sur le plan aube-à-aube}

Une caractéristique commune des répartitions de vitesse et turbulence sur les surfaces cylindriques analysées dans le paragraphe précédent est la tridimensionnalité non élevée de l'écoulement dans la première partie du diffuseur. Cette constatation laisse supposer qu'une analyse effectuée dans un plan aube-à-aube à mi-largeur d'aubage soit représentative de l'écoulement dans la région du diffuseur la plus intéressée par l'interaction avec les sillages d'aubage.

Les figures 4 et 5 montrent les répartitions du module de la vitesse absolue $\tilde{c}$ et de la turbulence $\overline{T u}$ sur un secteur annulaire compris entre $r / R_{2}=0,96$ et $r / R_{2}=1,26$ à 3 différents instants de temps appartenant à une période de passage d'aubage, soit 3 positions relatives des aubes de la roue et du diffuseur.

Pour étudier l'évolution de l'écoulement instationnaire dans le diffuseur, il peut être utile d'évaluer la fréquence réduite, définie par le rapport entre le temps que les particules emploient pour être transportées de l'entrée à la sortie du canal et la période de la perturbation, qui coïncide avec la période de passage des aubes. Ce rapport représente le nombre des sections de sillage qui sont contenues dans le canal du diffuseur à chaque instant. Si l'on applique cette définition à l'écoulement dans la région du diffuseur aubé où les mesures ont été effectuées, on obtient $\Omega f \Delta r / \bar{c}_{r}=1,96$.

L'évolution du sillage dans le diffuseur peut être étudiée en analysant les figures 4 et 5 . Le sillage est caractérisé par des niveaux élevés de vitesse absolue et de turbulence.
Si l'on observe le sillage de l'aube A du rotor, à l'instant $t / T_{i}=0,292$, il est centré dans l'anneau non aubé qui précède le canal 1-2 du diffuseur. A cet instant le col du canal 1-2 est traversé par un écoulement non perturbé à faible vitesse et turbulence. A l'instant suivant $t / T_{i}=0,492$ le sillage commence à être découpé par l'aube 1 du diffuseur et s'étend en direction azimutale sur toute la section d'entrée du diffuseur. Les niveaux de vitesse et turbulence sont peu changés en comparaison de l'instant précédent, le col du diffuseur est encore presque complètement libre d'écoulement perturbé. A l'instant $t / T_{i}=0,692$, un peu plus que la moitié du sillage résulte découpée par l'aube 1 , le col est presque complètement occupé par le sillage, la turbulence et la vitesse commencent à diminuer. Puisqu'on a limité les instants montrés sur les figures pour des raisons d'espace, l'analyse de l'évolution du sillage doit être poursuivie en examinant le canal 2-3 à l'instant $t / T_{i}=0,292$, où l'aube A se trouve par rapport à l'aube 2, presque dans la même position qu'elle aurait à l'instant $t / T_{i}=0,892$ par rapport à l'aube 1 . Dans cette condition l'aube 2 a complètement découpé le sillage et soit le taux de turbulence soit la vitesse commencent à diminuer significativement. Aux instants suivants $\left(t / T_{i}=0,492\right.$ et $t / T_{i}=$ 0,692 ) la section du sillage résulte "capturée " à l'intérieur du canal et soit la non-uniformité de vitesse soit le taux de turbulence diminuent rapidement.

Pour observer l'évolution du sillage vers la sortie de la région analysée du diffuseur, on peut retourner au canal 1-2, mais cette fois avec référence à la section de sillage issue de l'aube $\mathrm{B}$, à peine visible. Dans la dernière section de mesure $\left(r / R_{2}=1,26\right)$ l'écoulement est presque complètement mélangé et la variation azimutale de la vitesse est due principalement à la charge aérodynamique d'aubage.

\section{- 4.3 Ecoulement moyenné suivant le temps}

La figure 6 montre l'évolution dans la première partie du diffuseur de 3 grandeurs moyennées suivant le temps: la

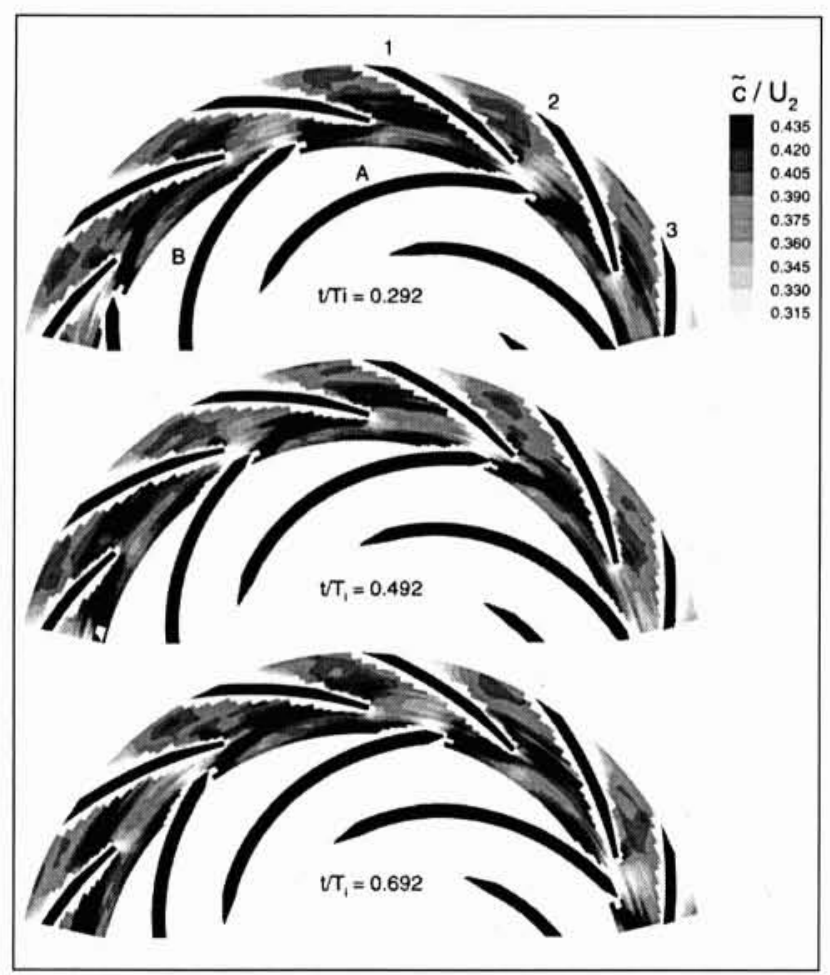

4. Séquence temporelle de la répartition de la vitesse absolue sur le plan de mesure aube-à-aube.

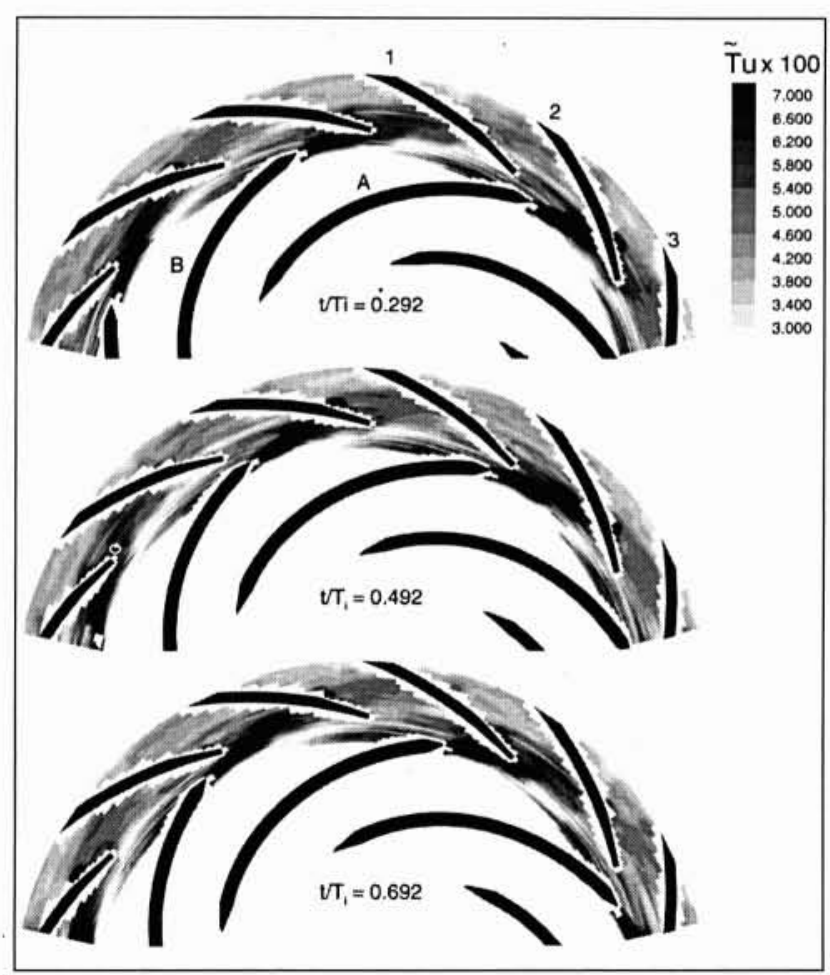

5. Séquence temporelle de la répartition du taux de turbulence sur le plan de mesure aube-à-aube. 
vitesse absolue, l'instationnarité générée par la roue et le taux de turbulence. La coordonnée azimutale $y$ est adimensionnalisée par le pas local du diffuseur $G_{d}=2 \pi r / z_{d}$ et augmente à partir du côté pression vers le côté dépression.

La distribution de la vitesse moyenne montre que, dans la première section de mesure, la charge aérodynamique est presque nulle et la diffusion très faible. Seulement après la section à $r / R_{2}=1,200$ on peut remarquer une diminution sensible de la vitesse sur le côté pression du canal. Par contre, la diminution à $r / R_{2}=1,048$, juste en amont du bord d'attaque des aubes, est due à l'effet de stagnation. Dans la section à $r / R_{2}=1,095$ on constate une augmenta-

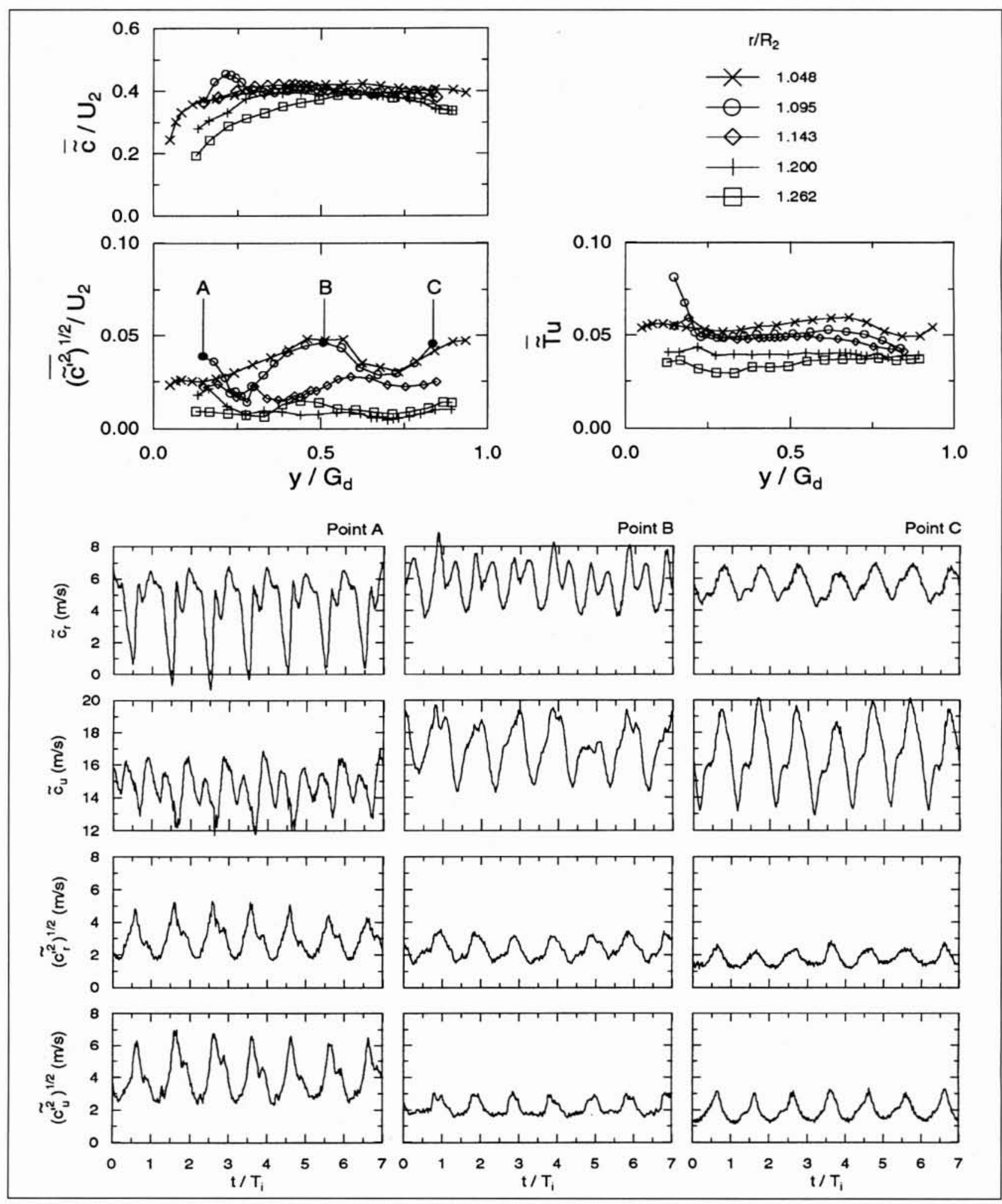

6. Evolution des répartitions azimutales de vitesse, instationnarité et taux de turbulence moyennés suivant le temps. Profils des composantes instantanées de vitesse et des valeurs r.m.s. des fluctuations turbulentes à quelques points de mesure $\left(r / R_{2}=1,095\right)$. 
tion localisée de la vitesse près du côté pression de l'aube, probablement liée à la faible incidence négative de l'écoulement.

Le coefficient d'instationnarité $\left(\overline{\tilde{C}^{12}}\right)^{1 / 2} / U_{2}$ et le taux de turbulence moyen $\widetilde{T u}$ dans les premières sections de mesure présentent des niveaux comparables et des valeurs élevées, supérieures à $5 \%(12 \%$ si on les rapporte à la vitesse absolue moyenne). L'instationnarité reste élevée dans la section de mesure proche du col du diffuseur $\left(r / R_{2}=1,095\right)$, mais diminue significativement dans les sections de sondage suivantes $\left(r / R_{2}=1,143\right)$. Par contre le taux de turbulence reste élevé jusqu'à $r / R_{2}=1,143$ et diminue seulement à partir de $r / R_{2}=1,200$. Ces répartitions suggèrent que l'atténuation de l'instationnarité soit le résultat non seulement du mélange convectif, mais aussi d'un processus de dissipation, qui se manifeste par le transfert d'énergie de l'écoulement organisé à la turbulence.

Dans la section $r / R_{2}=1,095$ les deux grandeurs montrent une augmentation près du côté pression de l'aube du diffuseur. Pour approfondir ce phénomène on peut analyser pour quelques points significatifs de cette section la répartition temporelle des composantes de la vitesse et des fluctuations turbulentes moyennées en phase. Le point $\mathrm{A}$ est situé sur le côté pression, $\mathrm{B}$ au milieu du canal et $\mathrm{C}$ sur le côté dépression (fig. 6).

L'instationnarité de l'écoulement dans la section $r / R_{2}=1,095$ proche du col du diffuseur est encore élevée et l'effet du diffuseur sur l'écoulement instationnaire est très important. En effet les profils des composantes de la vitesse changent d'un point à l'autre. En général le sillage, localisable autour des pics des valeurs r.m.s. des fluctuations turbulentes de la vitesse, est caractérisé par une augmentation de toutes les deux composantes moyennes de la vitesse. Au point $\mathrm{A}$ proche du côté pression, la présence de la paroi modifie sensiblement les profils des composantes de la vitesse, qui tout près des pics de turbulence montrent des minimums très marqués. Ce phénomène peut être expliqué par l'impact du jet négatif corrélé au sillage, qui cause la formation de tourbillons à contact de la paroi, qui pourraient induire le décollement instationnaire de la couche limite.

Les profils temporels des valeurs efficaces des fluctuations turbulentes révèlent que l'augmentation de la turbulence moyennée suivant le temps, remarquée précédemment près du côté pression, est due soit à l'élévation des pics des fluctuations turbulentes associés au passage du sillage soit à l'augmentation des valeurs de base.

\section{$\mathrm{V} \square \mathrm{CONCLUSIONS}$}

On a étudié l'écoulement instationnaire à l'entrée et à l'intérieur du diffuseur aubé d'un étage centrifuge. Les mesures effectuées sur deux surfaces cylindriques, situées juste en amont du bord d'attaque des aubes et près de la section d'entrée minimale, montrent un écoulement fortement instationnaire, dominé par le passage des sillages de la roue, mais caractérisé par une faible tridimensionnalité.

Dans la partie partiellement aubée du diffuseur, soit l'instationnarité générée par le rotor, soit la turbulence moyennée suivant le temps présentent des valeurs élevées, qui sont supérieures à $12 \%$ de la vitesse moyenne. Lorsqu'on dépasse le col du diffuseur, l'instationnarité diminue rapidement, tandis que la diminution du taux de turbulence moyen est plus lente, à cause du transfert d'énergie de l'écoulement instationnaire organisé aux fluctuations turbulentes.

L'écoulement dans le sillage est intéressé par une intense action convective dirigée du côté dépression vers le côté pression du canal du diffuseur, qui tend à concentrer sur le côté pression de l'aube l'écoulement caractérisé par une tur- bulence plus élevée. L'impact des sillages sur le côté pression des aubes du diffuseur cause des modifications significatives des profils temporels de la vitesse absolue, en induisant des pics de déficit des composantes de vitesse.

Des mesures détaillées de vitesse sur un plan aube-à-aube à mi-largeur d'aubage ont permis de reconstruire des images instantanées de l'écoulement pour différentes positions des aubes de la roue par rapport au diffuseur. Le sillage se manifeste comme une perturbation périodique, caractérisée par des valeurs élevées de la vitesse absolue et de la turbulence, qui se développe sur toute la zone non aubée et partiellement aubée du diffuseur qui précède le col d'entrée, avant d'être découpée par le bord d'attaque de l'aube en sections qui sont convectées à l'intérieur du canal.

Les résultats révèlent que le mélange du sillage et donc l'atténuation de l'instationnarité de l'écoulement dans le diffuseur en aval du col, déjà remarquée par d'autres rechercheurs, se manifeste plus rapidement après que le sillage a été découpé par l'aube du diffuseur et la portion de sillage a été isolée à l'intérieur du canal.

\section{BIBLIOGRAPHIE}

[1] INoue M., Cumpsty N.A. (1984). - « Experimental Study of Centrifugal Impeller Discharge Flow in Vaneless and Vaned Diffusers ». ASME Journal of Engineering for Gas Turbine and Power, vol. 106, pp. 455-467.

[2] Ubaldi M., Zunino P., Barigozzi G., Cattanei A. (1996). - « An Experimental Investigation of Stator Induced Unsteadiness on Centrifugal Impeller Outflow ». ASME Journal of Turbomachinery, vol. 118, pp. 41-54.

[3] Krain H. (1981). - «A Study on Centrifugal Impeller and Diffuser Flow ». ASME Journal of Engineering for Power, vol. 103, pp. 689-697.

[4] Toussaint M., Hureau F. (1993). - «Etude expérimentale de l'écoulement instationnaire dans une pompe centrifuge fonctionnant à débit partiel ». AIRH 6th International Meeting on The Behaviour of Hydraulic Machinery under Steady Oscillatory Conditions, Lausanne.

[5] Casey M.V., Eisele K., Zhang Z., Gülich J., SchachenMann A. (1996). - «Flow Analysis in a Pump Diffuser, Part 1: LDA and PTV Measurements of the Unsteady Flow ». FED-vol. 229, Laser Anemometry, ASME, pp. 89-100.

[6] Hodson H.P. (1985). - « Measurements of Wake-Generated Unsteadiness in the Rotor Passages of Axial Flow Turbines ». ASME Journal of Engineering for Gas Turbine and Power, vol. 107, pp. 467-476.

[7] ZacCaria M.A., LaKSHMinarayana B. (1995). - « Unsteady Flow Field Due to Nozzle Wake Interaction with the Rotor in an Axial Flow Turbine. Part I : Rotor Passage Flow Field ». ASME Paper 95-GT-295.

[8] Ubaldi M., Zunino P., Barigozzi G., Ghiglione A. (1996). - « LDV investigation of the rotor-stator aerodynamic interaction in a centrifugal turbomachine ». Eigth International Symposium on Applications of Laser Techniques to Fluid Mechanics, Lisbon.

[9] Rothe P.H., Johnston J.P. (1976). - «Effects of System Rotation on the Performance of Two-Dimensional Diffusers ». ASME Journal of Fluids Engineering, vol. 98, pp. $422-430$.

[10] MeYer R.X. (1958). - «The Effects of Wakes on the Transient Pressure and Velocity Distributions in Turbomachines ». ASME Journal of Basic Engineering, vol 80, pp. 1544-1552. 